\title{
AVALIAÇÃO DO ÍNDICE DE LANGELIER EM SISTEMA DE DISTRIBUIÇÃO DE ÁGUA DE SÃO CARLOS (SP), SUPRIDO POR MANANCIAL SUBTERRÂNEO: ESTUDO DE ESTABILIZAÇÃO
}

\author{
André Pina de Mesquita ${ }^{1}$
}

\author{
Erich Kellner ${ }^{2}$
}

\begin{abstract}
RESUMO
Um bairro do município de São Carlos (SP) tendo seu abastecimento de água feito manancial subterrâneo, através de um poço, apresentou reclamações sobre corrosão nas tubulações de cobre e placas de aquecimento solar. Foi observado que o índice de Langelier do poço era de $-4,5$ e que a água distribuída para região apresentada índice de Langelier de -1,74, após correção com $\mathrm{Na}_{2} \mathrm{CO}_{3}$. Desenvolveu-se estudo de estabilização da água para os agentes alcalinizantes $\mathrm{Na}_{2} \mathrm{CO}_{3}$ e $\mathrm{Ca}(\mathrm{OH})_{2}$. Para a faixa de pH estabelecida pela Portaria de Potabilidade 2914/2011, não foi possível estabilizar a água com $\mathrm{Na}_{2} \mathrm{CO}_{3} \mathrm{O}$ emprego do agente alcalinizante $\mathrm{Ca}(\mathrm{OH})_{2}$, com densidade 1,15, permitiu obter $\mathrm{IL}=+0,2$ com pH próximo a 9,0, mostrando-se adequado e mais econômico para a estabilização da água.
\end{abstract}

PALAVRAS-CHAVE: Corrosão. Índice de Langelier. Manancial subterrâneo.

\section{EVALUATION INDEX ASSESSMENT IN WATER DISTRIBUTION SYSTEM OF SÃO CARLOS (SP), MASTED IN STOCK UNDERGROUND: STABILIZATION OF STUDY}

\footnotetext{
${ }^{1}$ Aluno de graduação do curso de Engenharia Civil da Universidade Federal de São Carlos (UFSCar). E-mail: pinamesquita@gmail.com

${ }^{2}$ Engenheiro Civil pela Universidade Federal de São Carlos (UFSCar). Mestre e Doutor em Engenharia Civil: Area de Hidráulica e Saneamento pela Escola de Engenharia de São Carlos da Universidade de São Paulo (EESC/USP). Professor do Departamento de Engenharia Civil (DECiv) da Universidade Federal de São Carlos (UFSCar).E-mail: erich.kellner@ufscar.br
} 
GC

Revista Nacional de

Gerenciamento de Cidades

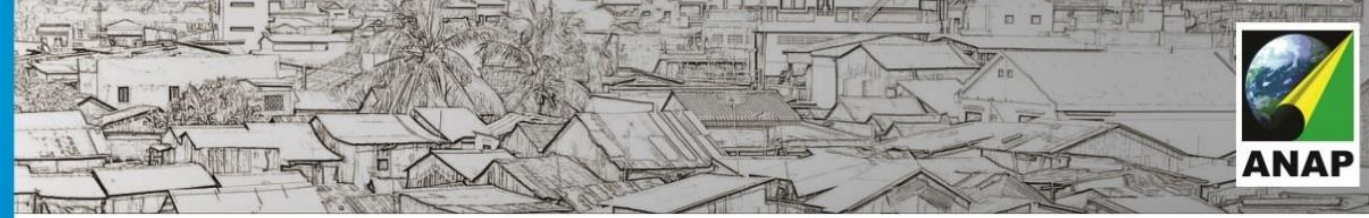

\begin{abstract}
A suburb of São Carlos (SP) having their water supply made underground spring, through a pit presented complaints about corrosion in copper pipes and solar heating plates. It was observed that groundwater's Langelier index was -4.5 and the water distributed to the region resulted Langelier index -1.74 after correction with $\mathrm{Na}_{2} \mathrm{CO}_{3}$. Study was developed for the stabilization of water $\mathrm{Na}_{2} \mathrm{CO}_{3}$ alkalizing agents. and $\mathrm{Ca}(\mathrm{OH})_{2}$. For the $\mathrm{pH}$ range established by Ordinance of potability 2914/2011, it was not possible to stabilize the water with $\mathrm{Na}_{2} \mathrm{CO}_{3}$. The use of alkalizing agent $\mathrm{Ca}(\mathrm{OH})_{2}$, with a density of 1.15, yielded $L=+0.2 \mathrm{pH}$ near 9.0, being suitable and more economical for the stabilization of the water.
\end{abstract}

KEYWORDS: Corrosion. Langelier Index. Groundwater

\title{
EVALUACIÓNÍNDICE DE EVALUACIÓN EN SISTEMA DE DISTRIBUCIÓN DE AGUA DE SÃO CARLOS (SP), MASTED EN STOCK UNDERGROUND: ESTABILIZACIÓN DE ESTUDIO
}

\begin{abstract}
RESUMEN
Un barrio de São Carlos (SP) que tiene su abastecimiento de agua realizado por manantial subterráneo, a través de un bien presentado quejas sobre la corrosión en las tuberías de cobre y placas solares térmicas. Se observó que el índice de Langelier bien era -4,5 y el agua distribuida a la región muestra índice de Langelier $-1,74$ después de la corrección con $\mathrm{Na}_{2} \mathrm{CO}_{3}$. Estudio fue desarrollado para la estabilización de los agentes alcalinizantes $\mathrm{Na} 2 \mathrm{CO} 3$ agua. y $\mathrm{Ca}(\mathrm{OH})_{2}$. Para el rango de $\mathrm{pH}$ establecido por la Ordenanza de potabilidad 2914/2011, no fue posible estabilizar el agua con $\mathrm{Na}_{2} \mathrm{CO}_{3}$. El uso de agente alcalinizante $\mathrm{Ca}(\mathrm{OH})_{2}$, con una densidad de 1,15 , produjo $\mathrm{L}=+$ $0,2 \mathrm{pH}$ cerca de 9,0, siendo adecuado y más económico para la estabilización del agua.
\end{abstract}

PALABRAS CLAVE: Corrosión. Índice de Langelier. aguas subterrâneas

\section{INTRODUÇÃO}

O uso dos mananciais subterrâneos vem se destacando como solução para o abastecimento de águas nos centros urbanos. Essa alternativa vem sendo adotada em regiões isoladas da malha urbana ou em setores urbanos que tiveram um aumento da demanda por água superior ao crescimento da infraestrutura de abastecimento. 
Revista Nacional de

Gerenciamento de Cidades

A qualidade físico-química das águas subterrâneas é função das interações com a mineralogia do solo/rocha durante sua infiltração e do seu tempo de permanência nos aquíferos além das influências antrópicas decorrentes do uso e ocupação do solo. Com isso é bastante comum encontrar-se águas com elevados índices de agressividade ou incrustação.

Uma das maneiras de se avaliar a agressividade das águas é a partir da avaliação do Índice de Saturação de Langelier, ou simplesmente, Índice de Langelier (IL), que representa um modelo de equilíbrio derivado conceito teórico de saturação da água relacionado ao carbonato de cálcio.

De maneira geral, no Brasil, o desenvolvimento dos sistemas de abastecimento de água parece não ter sido sustentado pelo aproveitamento dos mananciais subterrâneos, tanto que a legislação brasileira normalizadora dos recursos hídricos - Lei 9433/97 (BRASIL, 1997) não coloca as águas subterrâneas em um plano de destaque.

Nesse sentido, observa-se que as normas brasileiras relativas à qualidade das águas subterrâneas - Resoluções CONAMA 396 e 420 (BRASIL, 2008 e 2009), Portaria 2914 do Ministério da Saúde (BRASIL, 2011), não fazem referência às características de agressividade ou incrustação dessas águas. Com isso, tem-se observado casos em que, após a adequação aos padrões de potabilidade, a água distribuída mantém-se agressiva causando dados materiais aos usuários.

\section{OBJETIVO}

O objetivo do presente trabalho foi o de avaliar o Índice de Saturação de Langelier de um sistema de abastecimento de água devido ao aumento de ocorrências de problemas de corrosão observados nas instalações hidráulicas e propor procedimentos para estabilização da água. 
Revista Nacional de

Gerenciamento de Cidades

\section{MATERIAL E MÉTODOS}

Nesta seção são apresentados os materiais e métodos empregados para determinação do Índice de Saturação de Langelier e para condução dos estudos para estabilização da água.

\section{- Área de Estudo:}

O estudo foi realizado em um bairro do município de São Carlos (SP), Brasil, atendido por poço tubular com $200 \mathrm{~m}$ de profundidade e que captava água do aquífero Guarani, com vazão da ordem de $350 \mathrm{~m}^{3} / \mathrm{dia}$, e que apresentava várias ocorrências de corrosão em tubulações de cobre e placas de aquecimento solar.

A água proveniente do manancial subterrâneo era submetida a tratamento a fim de adequá-la aos padrões de potabilidade estabelecidos pela Portaria n. 2914/2011 do Ministério da Saúde (Brasil, 2011), sendo empregado $\mathrm{Na}_{2} \mathrm{CO}_{3}$ (barrilha leve) para elevação do pH, e $\mathrm{NaClO}$ (hipoclorito de sódio) para desinfecção, sendo posteriormente encaminhada ao reservatório elevado.

\section{- Determinação do Índice de Langelier}

O índice de saturação de Langelier (IL) é um modelo do equilíbrio derivado de conceitos teóricos de saturação que indica o grau de saturação do carbonato de cálcio na água, representado pela diferença entre o $\mathrm{pH}$ medido na água e $\circ \mathrm{pH}$ de saturação da água contendo íons como $\mathrm{Ca}^{+2}, \mathrm{Mg}^{+2}, \mathrm{HCO}_{3}$ quando o sistema $\mathrm{CO}_{3}^{-2} \mathrm{X}$ $\mathrm{CO}_{2}$ encontra-se em equilíbrio, conforme Equação (01).

$$
I L=p H-p H_{\text {saturaça } a}
$$

onde IL é o índice de Langelier (adimensional); $p H$ é o potencial hidrogeniônico da

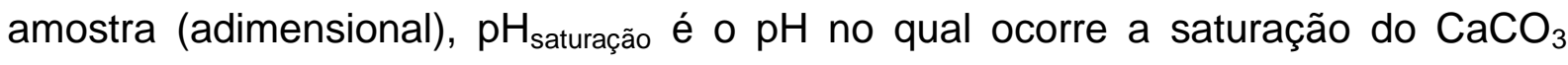
(adimensional).

$\mathrm{O} p \mathrm{H}_{\text {saturação }}$ pode ser obtido por coeficientes que dependem da temperatura da amostra, da alcalinidade, da dureza e da concentração de sólidos dissolvidos 
GC

Revista Nacional de

Gerenciamento de Cidades

totais, conforme método proposto por Langelier (1936) e representado pela Equação (02).

$$
p H_{\text {saturação }}=9,3+A+B-C-D
$$

onde $A, B, C, D$ são, respectivamente, parâmetros que dependem da concentração de sólidos dissolvidos totais (SDT), da temperatura (T) , da concentração de cálcio e da alcalinidade total da água amostrada, sendo expressos pelas Equações (03), (04), (05) e (06).

$$
\begin{aligned}
& A=\frac{\log (S D T)-1}{10} \\
& B=-13,12 \cdot \log (T+273)+34,55 \\
& C=\log \left(C a^{+2}\right) \\
& D=\log (A L K)
\end{aligned}
$$

sendo SDT a concentração de sólidos dissolvidos totais (mg/L); T a temperatura da água $\left({ }^{\circ} \mathrm{C}\right) ; \mathrm{Ca}^{+2}$ a concentração de cálcio como $\mathrm{CaCO}_{3}(\mathrm{mg} / \mathrm{L}) ; \mathrm{ALK}$ a alcalinidade como $\mathrm{CaCO}_{3}(\mathrm{mg} / \mathrm{L})$.

Consoante o valor para o índice de Langelier obtido, a água pode encontrarse em um dos três estados: em equilíbrio químico, quando $\mathrm{LSI}=0$; com tendência a ser corrosiva, quando $\mathrm{LSI}<0$; com tendência a ser encrustante, quando $\mathrm{LSI}>0$.

\section{- Determinação das amostras a serem analisadas}

Para realização das análises físico-químicas, foram coletados $5 \mathrm{~L}$ de água bruta, no cavalete do poço, e 0,5L de água na saída do reservatório de distribuição, sendo acondicionados em recipientes de vidro.

Antes do acondicionamento, abriram-se as torneiras de amostragem por 1 minuto. Em seguida, procedeu-se ao tríplice enxague e, posteriormente, procedeuse a coleta dos volumes amostrados. 


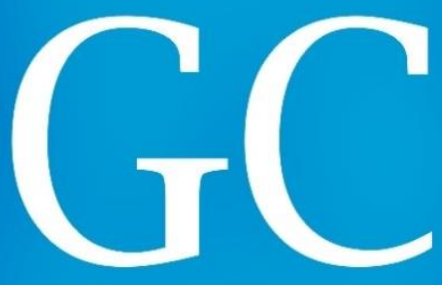

Revista Nacional de

Gerenciamento de Cidades

- Ensaios para caracterização físico-química das amostras coletadas e para condução dos estudos de estabilização da água

Para determinação do Índice de Lagelier das amostras de água analisadas, foram determinadas as variáveis constantes da Tabela 1.

Tabela1: Metodologias analíticas para medição de variáveis empregadas na determinação do Índice de Langelier

\begin{tabular}{llccc}
\hline \multicolumn{1}{r}{ Variáveis Analisadas } & Unidade & \multicolumn{1}{c}{ Método } & $\begin{array}{c}\text { Referência } \\
\text { Bibliográfica }\end{array}$ \\
\hline 1 & Temperatura & ${ }^{\circ} \mathrm{C}$ & potenciométrico & --- \\
2 & $\mathrm{pH}$ & --- & potenciométrico & -- \\
3 & Alcalinidade & $\mathrm{mg} / \mathrm{L}$ & Titulação potenciométrica & APHA (1995) \\
4 & Dureza & $\mathrm{mg} / \mathrm{L}$ & Titulação potenciométrica & APHA (1995) \\
5 & Sólidos Dissolvidos Totais & $\mathrm{mg} / \mathrm{L}$ & Gravimetria & APHA (1995) \\
\hline
\end{tabular}

Com relação ao estudo de estabilização da água, considerou-se dois tipos de agentes alcalinizantes: a barrilha leve $\left(\mathrm{Na}_{2} \mathrm{CO}_{3}\right)$ e solução hidróxido de cálcio $\left(\mathrm{Ca}(\mathrm{OH})_{2}\right)$ com densidade de 1,15.

Para o estudo de estabilização da água, tomou-se, para cada agente alcalinizante foi considerada, o volume de $200 \mathrm{~mL}$ de água bruta proveniente do poço, sendo introduzido um volume da solução alcalinizante correspondente, de maneira a obter valores de pH de 7,0, 7,5, 8,0, 8,5 e 9,0.

Após a introdução dos agentes alcalizantes às amostras de água bruta, manteve-se as amostras em agitação, com auxílio de agitador magnético, durante 1 minuto, a fim de homogeneizar o volume a ser analisado.

Para cada um desses volumes que receberam diferentes doses de agentes alcalinizantes foram determinados os índices de Langelier. 
GC

Revista Nacional de

Gerenciamento de Cidades

\section{RESULTADOS}

Os resultados obtidos para a caracterização físico-química da água proveniente do poço e da saída do reservatório de distribuição estão apresentados na Tabela 2.

Tabela2: Características físico-químicas da água proveniente do poço e da saída do reservatório de distribuição

\begin{tabular}{cccccc}
\hline Amostra & pH & $\begin{array}{c}\text { Temperatura } \\
\left({ }^{\circ} \mathbf{C}\right)\end{array}$ & $\begin{array}{c}\text { Alcalinidade } \\
(\mathbf{m g} / \mathbf{L})\end{array}$ & $\begin{array}{c}\text { Dureza } \\
(\mathbf{m g} / \mathbf{L})\end{array}$ & $\begin{array}{c}\text { Sólidos } \\
\text { Dissolvidos Totais } \\
(\mathbf{m g} / \mathbf{L})\end{array}$ \\
\hline Água Bruta & 5,6 & 22 & 6,0 & 7,0 & 24 \\
Água Distribuida & 7,0 & 22 & 16 & 7,0 & 13 \\
\hline
\end{tabular}

Os resultados obtidos para as características físico-químicas da água proveniente do poço após a realização do ensaio de estabilização empregando $\mathrm{Na}_{2} \mathrm{CO}_{3}$ e $\mathrm{Ca}(\mathrm{OH})_{2}$ estão apresentados, respectivamente, nas Tabelas 3 e 4.

Tabela3: Características físico-químicas da água proveniente do poço após ensaio de estabilização empregando $\mathrm{Na}_{2} \mathrm{CO}_{3}$

\begin{tabular}{ccccccc}
\hline Amostras & $\begin{array}{c}\text { Concentração } \\
\mathrm{Na}_{2} \mathrm{CO}_{3} \text { na } \\
\text { amostra } \\
(\mathrm{mg} / \mathrm{L})\end{array}$ & $\mathrm{pH}$ & $\begin{array}{c}\text { Temperatura } \\
\left({ }^{\circ} \mathrm{C}\right)\end{array}$ & $\begin{array}{c}\text { Alcalinidade } \\
(\mathrm{mg} / \mathrm{L})\end{array}$ & $\begin{array}{c}\text { Dureza } \\
(\mathrm{mg} / \mathrm{L})\end{array}$ & $\begin{array}{c}\text { Sólidos } \\
\text { Dissolvidos Totais } \\
(\mathrm{mg} / \mathrm{L})\end{array}$ \\
\hline 1 & 32 & 7,0 & 22 & 21,0 & 7,0 & 35 \\
2 & 37 & 7,5 & 22 & 30,0 & 7,0 & 39 \\
3 & 46 & 8,0 & 22 & 40,0 & 7,0 & 45 \\
4 & 53 & 8,5 & 22 & 54,0 & 7,0 & 63 \\
5 & 74 & 9,0 & 22 & 74,0 & 7,0 & 69 \\
\hline
\end{tabular}




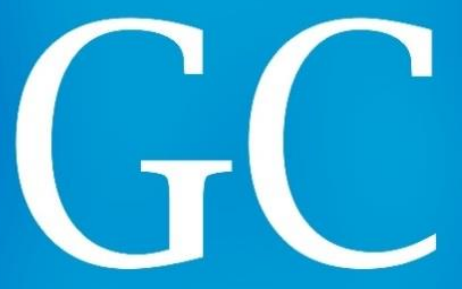

Revista Nacional de

Gerenciamento de Cidades

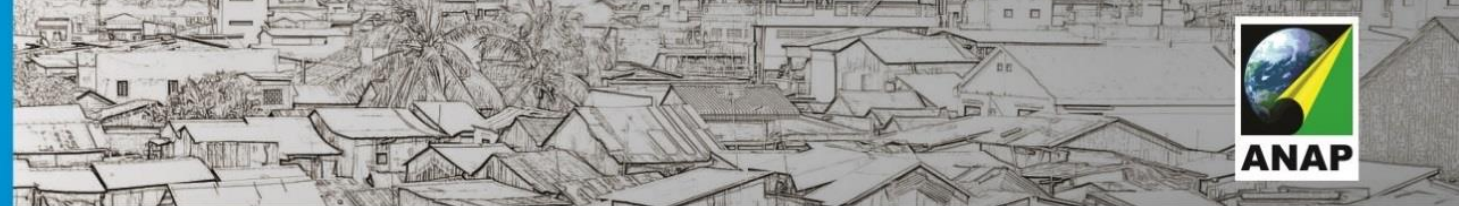

Tabela4: Características físico-químicas da água proveniente do poço após ensaio de estabilização empregando $\mathrm{Ca}(\mathrm{OH})_{2}$

\begin{tabular}{ccccccc}
\hline Amostras & $\begin{array}{c}\text { Concentração } \\
\mathrm{Ca}(\mathrm{OH})_{2} \text { na } \\
\text { amostra } \\
(\mathrm{mg} / \mathrm{L})\end{array}$ & $\mathrm{pH}$ & $\begin{array}{c}\text { Temperatura } \\
\left({ }^{\circ} \mathrm{C}\right)\end{array}$ & $\begin{array}{c}\text { Alcalinidade } \\
(\mathrm{mg} / \mathrm{L})\end{array}$ & $\begin{array}{c}\text { Dureza } \\
(\mathrm{mg} / \mathrm{L})\end{array}$ & $\begin{array}{c}\text { Sólidos } \\
\text { Dissolvidos Totais } \\
(\mathrm{mg} / \mathrm{L})\end{array}$ \\
\hline 1 & 33,3 & 7,5 & 22 & 18 & 15 & 44 \\
2 & 43,7 & 8,0 & 22 & 22 & 20 & 62 \\
3 & 72,8 & 9,0 & 22 & 30 & 29 & 73 \\
4 & 106,1 & 9,5 & 22 & 39 & 42 & 95 \\
\hline
\end{tabular}

Os valores do $\mathrm{pH}$ de saturação, assim como os Índices de Langelier obtidos para as amostras analisadas estão apresentados na Tabela 5.

Tabela 5: Resultados obtidos para o $\mathrm{pH}_{\text {saturacaao }}$ e para o Índice de Langelier para as várias amostras analisadas

\begin{tabular}{|c|c|c|c|c|c|c|}
\hline \multicolumn{2}{|c|}{ Amostra } & $\begin{array}{c}\text { Concentração } \\
\mathrm{Na}_{2} \mathrm{CO}_{3} \\
(\mathrm{mg} / \mathrm{L})\end{array}$ & $\begin{array}{c}\text { Concentração } \\
\mathrm{Ca}(\mathrm{OH})_{2} \\
(\mathrm{mg} / \mathrm{L})\end{array}$ & pH & $\mathrm{pH}_{\text {saturação }}$ & $\begin{array}{c}\text { Índice de } \\
\text { Langelier - } \\
\text { IL }\end{array}$ \\
\hline \multicolumn{2}{|c|}{ Água Bruta } & ---- & ---- & 5,6 & 10,1 & $-4,5$ \\
\hline \multicolumn{2}{|c|}{ Água distribuida } & ---- & ---- & 7,0 & 9,7 & $-2,7$ \\
\hline \multirow{5}{*}{ 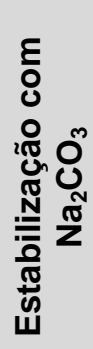 } & 1 & 32 & ---- & 7,0 & 9,6 & $-2,6$ \\
\hline & 2 & 37 & ---- & 7,5 & 9,4 & $-1,9$ \\
\hline & 3 & 46 & ----- & 8,0 & 9,3 & $-1,3$ \\
\hline & 4 & 53 & ----- & 8,5 & 9,2 & $-0,7$ \\
\hline & 5 & 74 & ----- & 9,0 & 9,1 & $-0,1$ \\
\hline \multirow{4}{*}{ 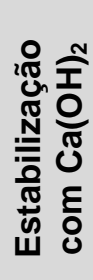 } & 1 & ---- & 33,3 & 7,5 & 9,7 & $-2,7$ \\
\hline & 2 & ----- & 43,7 & 8,0 & 9,1 & $-0,6$ \\
\hline & 3 & ---- & 72,8 & 9,0 & 8,85 & $+0,02$ \\
\hline & 4 & ----- & 106,1 & 9,5 & 8,59 & $+0,91$ \\
\hline
\end{tabular}




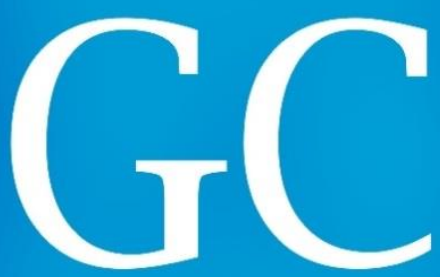

Revista Nacional de

Pela análise dos resultados apresentados na Tabela 4 é possível verificar que o índice de Langelier para a água de distribuição resultou em -2,7, ou seja, com potencial moderado para corrosão. Da mesma maneira, ao se aplicar $\mathrm{Na}_{2} \mathrm{CO}_{2} \mathrm{como}$ agente alcalizante, em concentração suficiente para manter o pH 9,0, obteve-se $\mathrm{IL}=$ 0,1 , enquanto ao se aplicar $\mathrm{Ca}(\mathrm{OH})_{2}$ em concentração suficiente para manter $\mathrm{opH}$ em 9,0, obteve-se IL $=+0,02$.

As Figuras 1 e 2 ilustram a redução do Índice de Langelier obtida a partir do aumento do $\mathrm{pH}$ proporcionado pelo aumento da concentração de $\mathrm{Na}_{2} \mathrm{CO}_{3}$ e $\mathrm{Ca}(\mathrm{OH})_{2}$, empregados respectivamente, como agente alcalinizantes, nos ensaios de estabilização.

Figura 1: Variação do Índice de Langelier (IL) em função do pH obtido após estabilização da água bruta empregando $\mathrm{Na}_{2} \mathrm{CO}_{3}$ como agente alcalinizante

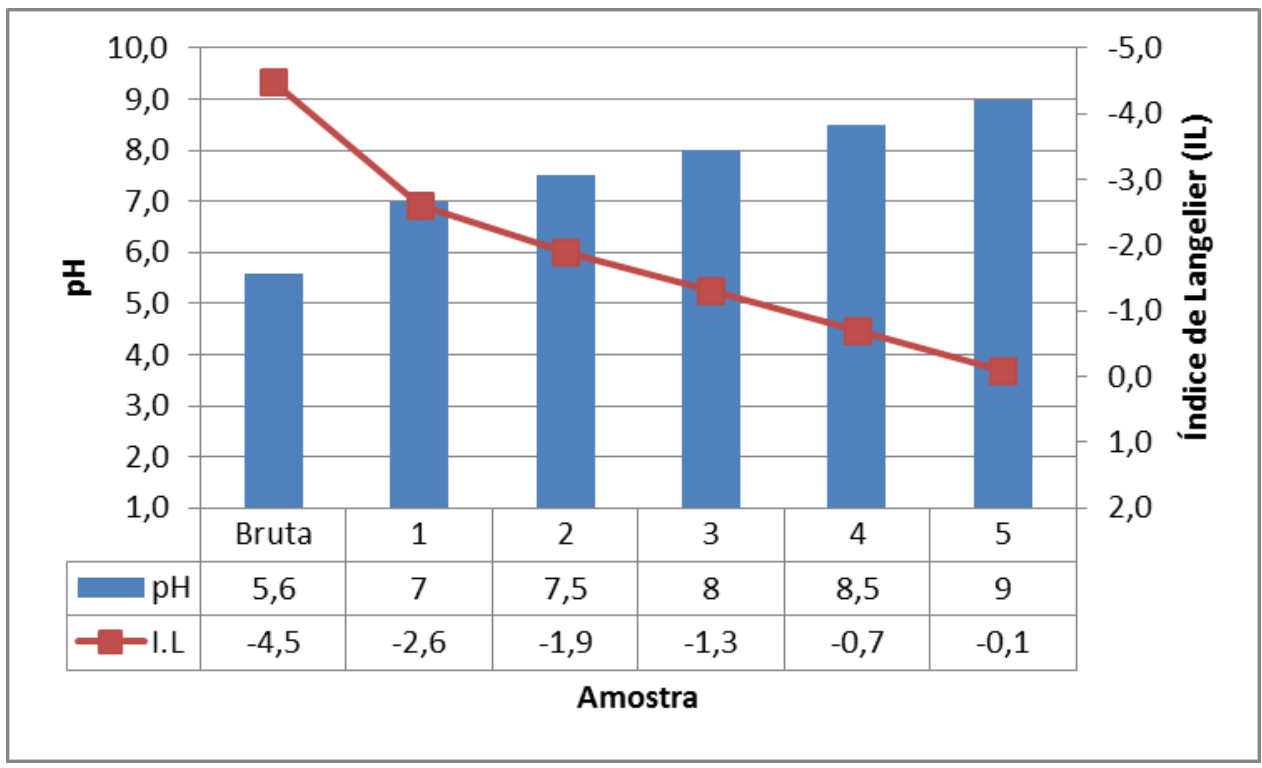

Fonte: os autores. 


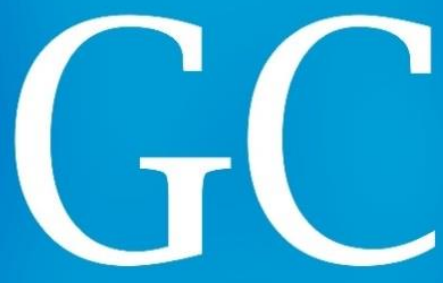

Revista Nacional de

Gerenciamento de Cidades

Figura 2: Variação do Índice de Langelier (IL) em função do pH obtido após estabilização da água bruta empregando $\mathrm{Ca}(\mathrm{OH})_{2}$ como agente alcalinizante

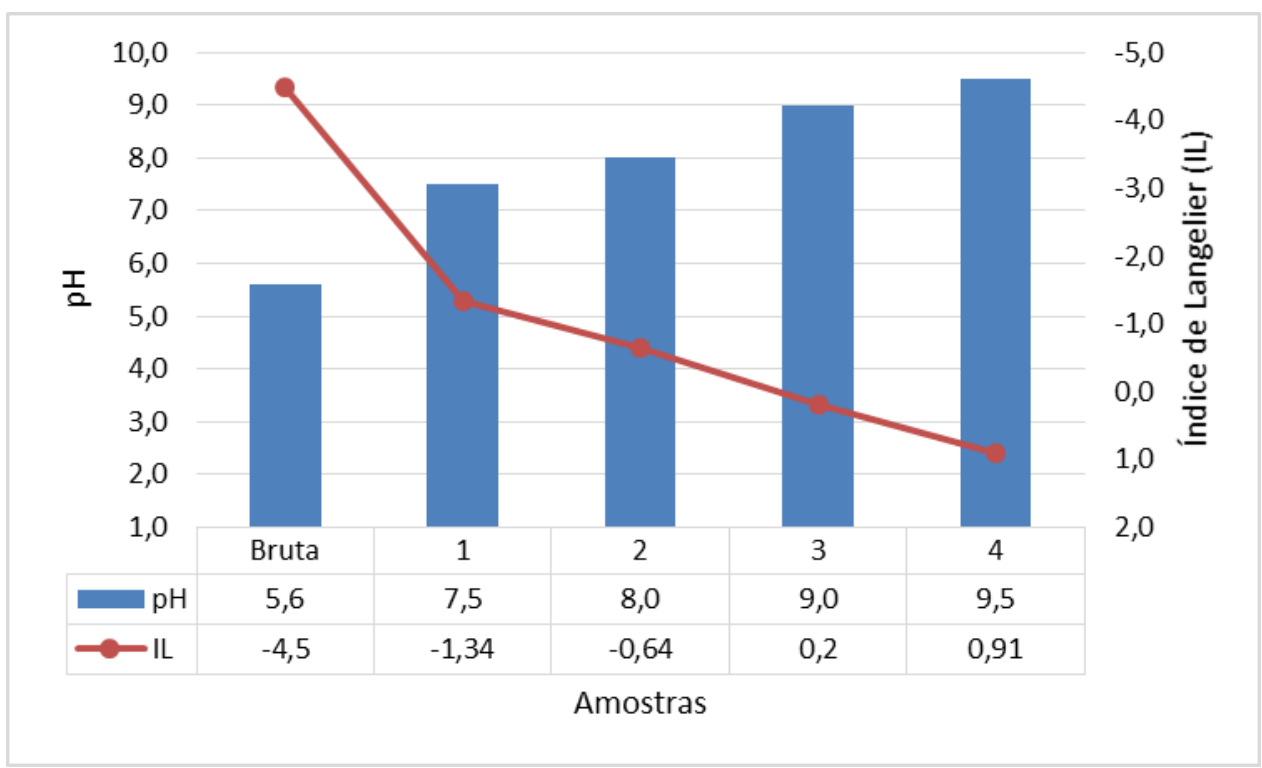

Fonte: os autores.

Considerando o pH de 9,0 para água estabilizada e a produção média do poço de $350 \mathrm{~m}^{3} / \mathrm{dia}$, estimou-se os volumes necessários para cada um dos agentes alcalinizantes empregados, estando os resultados apresentados na Tabela 6.

Tabela 6: Estimativa dos quantitativos e custos observados para aplicação dos agentes alcalinizantes avaliados no estudo

\begin{tabular}{lccccr}
\hline $\begin{array}{c}\text { Agente } \\
\text { Alcalinizante }\end{array}$ & $\begin{array}{c}\text { Concentração } \\
\text { considerada } \\
(\mathrm{mg} / \mathrm{L})\end{array}$ & $\mathrm{IL}$ obtido & $\begin{array}{c}\text { Quantidade } \\
\text { necessária } \\
(\mathrm{kg} / \mathrm{dia})\end{array}$ & $\begin{array}{c}\text { Custo } \\
\text { Unitário* } \\
(\mathrm{R} \$ / \mathrm{kg})\end{array}$ & $\begin{array}{r}\text { Custo Médio } \\
\text { Mensal para } \\
\text { estabilização } \\
(\mathrm{R} \$)\end{array}$ \\
\hline $\mathrm{Na}_{2} \mathrm{CO}_{3}$ & 74,0 & $-0,1$ & 25,9 & 2,25 & 1748,25 \\
$\mathrm{Ca}(\mathrm{OH})_{2}$ & 72,8 & $+0,2$ & 25,5 & 0,60 & 459,00 \\
\hline
\end{tabular}

Nota: * os custos unitários foram baseados em informações apuradas juntos aos fornecedores locais, considerando-se as quantidades mensais necessárias a estabilização da água. 
Revista Nacional de

Gerenciamento de Cidades

\section{CONCLUSÕES E RECOMENDAÇÕES}

A água proveniente do manancial subterrâneo apresentou $\mathrm{pH}$ de 5,6. pH de saturação de 10,1, resultando no Índice de Langelier de -4,53, caracterizando-a como água agressiva, com elevado poder de corrosão.

A água proveniente do reservatório de distribuição, após condicionamento adotado para adequação aos padrões de potabilidade estabelecidos pela Portaria $\mathrm{n}$. 2914/11 (Brasil, 2011) apresentou pH de 7,0, pH de saturação de 9,7 e Índice de Langelier de -2,7, caracterizando-a como agressiva com elevado poder de corrosão.

Após o estudo de estabilização da água empregando $\mathrm{Na}_{2} \mathrm{CO}_{3}$ indicou a necessidade de se operar com concentração de $74 \mathrm{mg} / \mathrm{L}$ a resultando $\mathrm{pH}$ de $9,0, \mathrm{pH}$ de saturação de 9,1 e Índice de Langelier de -0,1, alterando a caracterização da água para levemente agressiva. Em função de o pH obtido após a estabilização $(9,0)$ estar próximo do limite de potabilidade sugerido $(9,5)$ procedeu-se ao estudo de estabilização empregando-se $\mathrm{Ca}(\mathrm{OH})_{2}$ como agente alcalinizante.

$\mathrm{O}$ estudo de estabilização da água empregando $\mathrm{Ca}(\mathrm{OH})_{2}$ indicou a necessidade de se operar com concentraçãos próximas a $72 \mathrm{mg} / \mathrm{L}$, de maneira a resultar $\mathrm{pH}$ de 9,0, $\mathrm{pH}$ de saturação de 8,9 e Índice de Langelier de $+0,1$, muito próximo da condição de neutralidade (IL=0).

A substituição do agente alcalinizante para hidróxido de Cálcio $\left(\mathrm{Ca}(\mathrm{OH})_{2}\right)$, além de permitir a obtenção de Índice de Langelier próximo a zero, dentro dos limites de potabilidade estabelecidos pela Portaria 2914/2011 do Ministério da Saúde (BRASIL, 2011), resultou um custo médio mensal inferior quando se comparado com o agente alcalinizante $\mathrm{Na}_{2} \mathrm{CO}_{3}$ que vinha sendo empregado.

Seria pertinente que na revisão da Portaria 2914/11 fosse discutido a inclusão de indicadores para avaliar a agressividade e/ou incrustação das águas. 


\section{REFERÊNCIAS BIBLIOGRÁFICAS}

AMERICAN PUBLICH HEALTH ASSOCIATION - APHA. Standard Method for the examination of water and wastewater. 19ed. Washignton: Byrd Prepess Springfield, 1995.

BRASIL. Ministério da Saúde. Secretaria de Vigilância em Saúde. Portaria no 2914/11 de 12 de dezembro de 2011. Dispõe sobre vigilância e controle da qualidade da água para consumo humano. Brasília, 2011.

BRASIL. Conselho Nacional do Meio Ambiente - CONAMA. Resolução no 420/09 de 30 de dezembro de 2009. Dispõe sobre critérios e valores orientadores de qualidade de solo e águas subterrâneas quanto à presença substâncias químicas. Brasília, 2009

BRASIL. Conselho Nacional do Meio Ambiente - CONAMA. Resolução no 396/08 de 03 de abril de 2008. Dispõe sobre a classificação e diretrizes ambientais para o enquadramento das águas subterrâneas e dá outras providências. Brasília, 2008.

BRASIL. Presidência da República. Lei no $9433 / 97$ de 08 de janeiro de 1997. Institui a Política Nacional de Recursos Hídricos, cria o Sistema Nacional de Gerenciamento de Recursos Hídricos. Brasília, 1997.

LANGELIER, W. F., The Analytical Control of Anticorrosion Water Treatment. Journal of American Water Works Association 1936, 28, 1500-1521. 